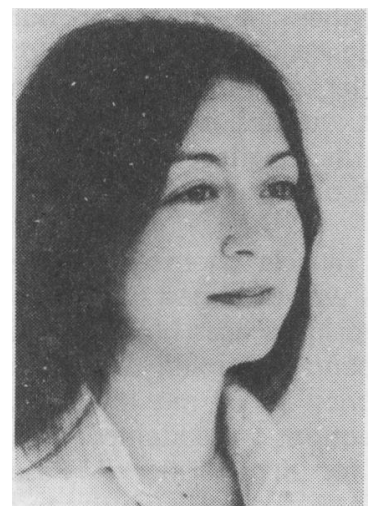

Michelle Mottola

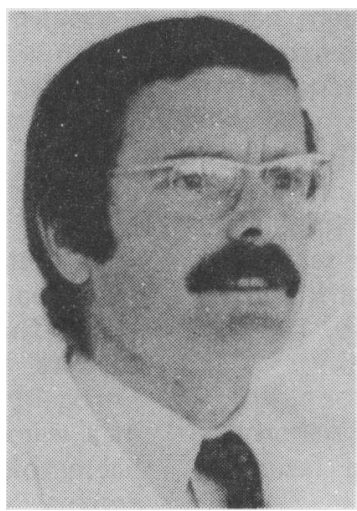

K. M. Bagnall

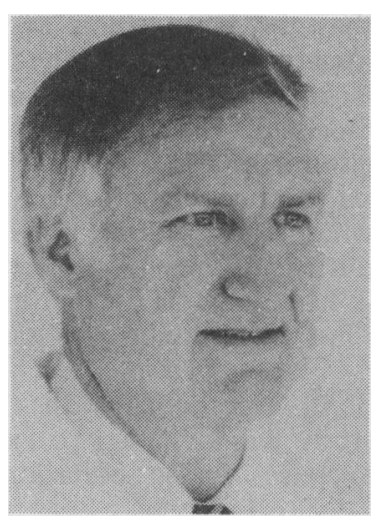

K. D. McFadden

\title{
THE EFFECTS OF MATERNAL EXERCISE ON DEVELOPING RAT FETUSES
}

\author{
Michelle MOTTOLA, MSc, K. M. BAGNALL, PhD and K. D. McFADDEN, PhD
}

Department of Anatomy, University of Alberta, Edmonton, Alberta, Canada

\begin{abstract}
Ten female Sprague-Dawley rats $(45.3$ days old, s.d. $=3.9$ ) were acclimatised to running on a treadmill at $20 \mathrm{~m} / \mathrm{min}$ on a $10^{\circ}$ incline for 1 hour per day. After mating, one rat from each of 5 pairs continued running on the treadmill at $15 \mathrm{~m} / \mathrm{min}$ on a $10^{\circ}$ incline for 1 hour per day throughout gestation whilst the other rats followed normal laboratory routine. All the rats were weighed daily and at birth the number and weights of all fetuses were measured. In addition, a small piece of diaphragmatic muscle was taken from each of three fetuses from each litter and assessed both histologically and histochemically.

The results showed that the maternal rats that did not continue exercising throughout pregnancy produced, on average, approximately 3 more fetuses per litter than the rats that exercised, although this difference was not significant. There was also no difference in mean fetal weight between the two groups and no difference could be demonstrated in the development of the diaphragm muscle.
\end{abstract}

\section{INTRODUCTION}

The effects of maternal exercise on fetal well-being are largely unknown (Clapp, 1980). This is surprising because maternal exercise has been shown to affect the fetus. For example, fetal breathing movements have increased after maternal exercise of short duration (Marsal et al, 1979). Further it is possible that this increased fetal activity may aid in fetal maturation, for Wirsen and Larsson (1964) suggested that maturation at birth is dependent on the length of gestation and motor activity of the fetus.

It appears reasonable to suggest that the by-products of maternal activity will alter the uterine environment which plays an important role in the development of the fetus. Even small changes in this environment can affect the fetus acutely for Lewis and Boylan (1979) found that a small amount of alcohol ingested by a woman during pregnancy leads to a decrease in fetal breathing movements, presumably by depressing the central nervous system. Similarly, alterations in the uterine environment induced by maternal smoking, amniocentesis, and maternal fasting have also been shown to affect the fetus acutely by reducing body movements. It is of additional interest that fetal body movements have been shown to be good indicators of fetal well-being (Pomerance et al, 1974; Patrick, 1977; Gettinger et al, 1978).

The accumulative effects on the fetus of these continuous or repeated changes in the uterine environment during gestation are not known, although maternal smoking throughout pregnancy has been shown to reduce birth weight (Gennser et al, 1976). It is possible 
that the by-products of maternal exercise might have long-term effects on the fetus particularly if the exercise is of high intensity, long duration, and frequently repeated. The redistribution of blood flow during exercise might also have an effect on the fetus by influencing nutrition and gaseous exchange, although it is not clear whether the blood flow to the placenta is impaired during exercise (Woodward, 1981). After exercise, there is a hyperaemia of the uterus which could also affect the fetus by producing a "tidal" effect (Woodward, 1981).

In accordance with this lack of information, this study was designed to examine the effects of maternal exercise during gestation on the developing fetuses.

\section{METHOD}

Fifteen young female Sprague-Dawley rats (mean age 45.3 days, s.d. $=3.9$; mean weight $155.5 \mathrm{~g}$, s.d. $=23.2$ ) were acclimatised to running on a treadmill at a speed of $20 \mathrm{~m} / \mathrm{min}$ on a $10^{\circ}$ incline for periods of one hour each day. This was accomplished using a progressive schedule which lasted for 18 days. This was considered the shortest time period needed by the rats to become accustomed to the exercise routine and was not intended to be a training period. Any training effect was assumed to be minimal. Previous experiments had shown that without this acclimatisation period the rats are unable to cope with the required exercise throughout pregnancy. They were then divided into three groups after this acclimatisation period. Ten of the rats were then paired by weight and housed together for the remainder of the experiment. Each pair was mated with one male over a four day period to ensure pregnancy (oestrus cycle $=4$ days). After the mating period, one female from each pair continued to exercise on the treadmill for 1 hour per day at a reduced rate of $15 \mathrm{~m} / \mathrm{min}$ on a $10^{\circ}$ incline throughout the whole gestational period. This group was called the "pregnant running" group (PR). The rate was reduced from the $20 \mathrm{~m} / \mathrm{min}$ to ensure that the rats would be able to cope without undue difficulty in the later stages of pregnancy. A comparison with the workloads used by other workers (Parizkova, 1975) suggests that the exercise routine used was of a fairly mild aerobic character throughout gestation.

The remaining pregnant females (the other from each pair) acted as a control group undertaking no additional exercise other than that of a normal laboratory routine. This group was called the "pregnant control" group (PC).

During the study all the rats were weighed each day prior to running and were fed and watered ad libitum.

At birth, the neonatal rats were counted and weighed and the three largest fetuses from each litter were selected for further analysis. These fetuses were selected for it is unknown whether larger fetuses have greater muscular development than smaller ones. However, our previous work (unpublished) suggested that the stage of muscular development was similar in all fetuses from the same litter regardless of size. It was not possible in this study to select fetuses of the same weight from different litters for the mothers gave birth on different days.

A section of diaphragmatic muscle taken each time from the same right costal area was dissected from each fetus. The section was mounted in an agar block, fixed to cork and immersed in isopentane cooled by liquid nitrogen. The block was sectioned at $10 \mathrm{mu}$ thickness in a cryostat at $-25^{\circ} \mathrm{C}$. The sections were stained with haematoxylin and eosin and for myofibrillar ATPase activity with pre-incubation at $10.4 \mathrm{pH}$ (Guth and Samaha, 1969) to study the histological and histochemical features.

A black and white photograph was taken of each section and from this several observations were made: the number of rosettes, the number of beta fibres, the number of alpha fibres, the average size of beta and alpha fibres, and the amount of connective tissue. This was an attempt to assess the maturity of the muscle according to the development sequence as outlined by Ashmore et al (1972).

Two control groups were run concurrently with the experiment. One group consisted of the 5 female Sprague-Dawley rats who had undertaken the same acclimatisation schedule as groups PR and PC. After this acclimatisation period they stopped running on the treadmill and were not mated, being allowed to develop normally. This was designed to determine the effects of the acclimatisation schedule on the mothers, as other workers (Dohm and Beecher, 1981) have found that greater work loads, which constitute training, lead to reduced weight. Although our schedule was not designed as a training programme it was thought desirable to assess its effect on normal development. The other group consisted of five female Sprague-Dawley rats who were also approximately $\mathbf{4 5}$ days old and who were allowed to develop normally following routine laboratory procedure. They were weighed each day and a chart of normal non-pregnant growth was produced.

Comparisons were made between the various measurements taken using the Student's " $t$ " test and the Mann-Whitney $U$ test where appropriate. The level of significance was chosen as $p<0.05$.

\section{RESULTS}

Figure 1 shows the mean weight gain for each of the four groups of rats during the period of gestation. It is of interest that the control group of pregnant rats (PC) has consistently gained more weight than that of the pregnant exercised rats (PR). This difference is present 
from the beginning of the gestational period and gets gradually wider as pregnancy progresses.

Also shown in Figure 1 are the mean number of fetuses born to the PC and PR groups. There is a mean difference of approximately 3 fetuses per litter but this is not statistically significant. Coupled with this, the mean weights of all the fetuses from the PC and PR groups are very similar which suggests that the difference in weight gain between the two groups throughout gestation can be attributed mainly to the difference in the number of fetuses (mean fetal weights: $P C=7.0 \mathrm{~g}$ s.d. $=0.4, P R=7.3 \mathrm{~g}$, s.d. $=1.2$ ).

Figure 2 shows a typical photograph used to assess the maturity of the diaphragm muscle. Rosettes, alpha fibres and beta fibres can clearly be seen and these were used in the assessment. The results are shown in Table I and no significant differences were found in the parameters measured between the PC and PR groups.

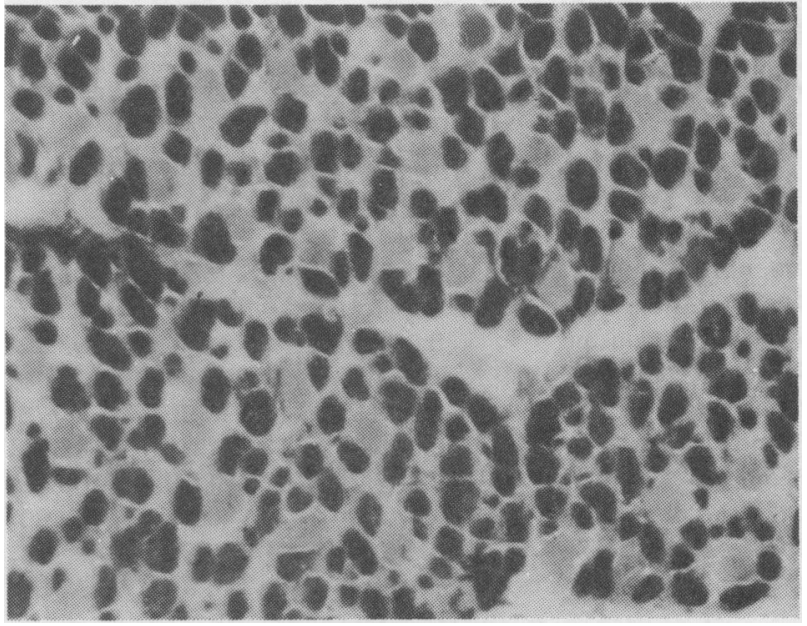

Fig. 2: An example of fetal diaphragm muscle. Alpha fibres are dark and beta fibres are light.

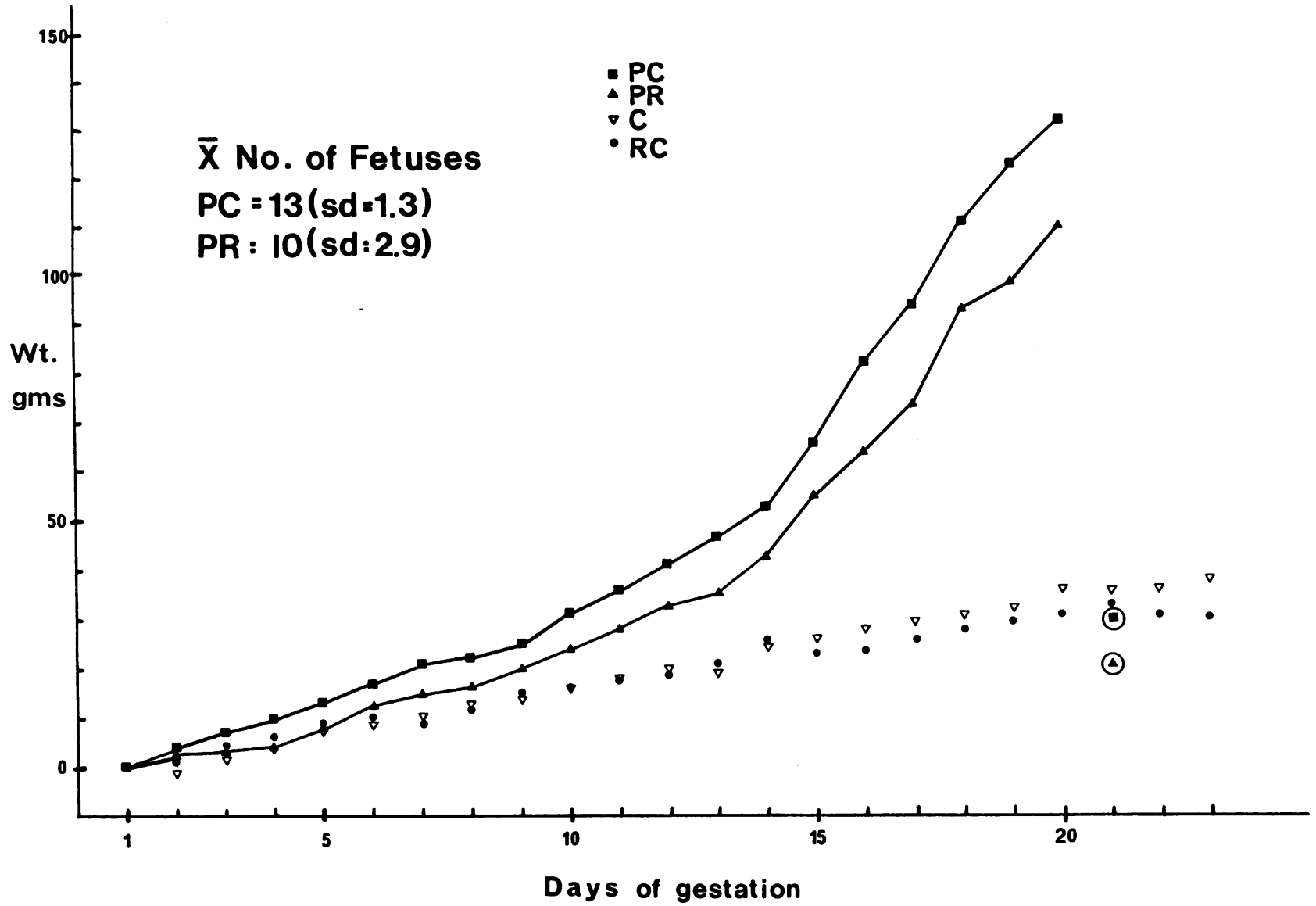

Fig. 1: The daily weight gain for each of the four groups of rats. Also indicated are the mean number of fetuses for the $P C$ and $P R$ groups. 


\section{TABLE I}

Analysis of fetal diaphragm muscle. Mean values and s.d. in brackets.

\begin{tabular}{lrcc} 
& \multicolumn{2}{c}{ Group } & P \\
& PC & PR & Value \\
No. of beta fibres & $18.5(4.6)$ & $19.3(5.2)$ & 0.803 \\
No. of clusters & $1.0(1.4)$ & $0.9(1.0)$ & 0.887 \\
Alpha fibres/cluster & $8.0(0.8)$ & $7.7(1.3)$ & 0.672 \\
Alpha fib. with vacuoles & $1.8(4.0)$ & $10.5(14.9)$ & 0.246 \\
No. clusters with vacs. & $0.4(8.9)$ & $0.3(0.5)$ & 0.775 \\
\% connective tissue & $39.8(8.2)$ & $42.7(11.7)$ & 0.662 \\
\% muscle & $60.4(7.7)$ & $57.2(11.7)$ & 0.623 \\
Diameter beta fibres mu & $4.8(0.6)$ & $5.1(0.4)$ & 0.380 \\
Diameter alpha fibres mu & $3.9(0.3)$ & $4.0(0.6)$ & 0.748 \\
\% alpha fibres with vacs. & $1.1(2.5)$ & $11.3(9.9)$ & 0.058
\end{tabular}

\section{DISCUSSION}

Woodward (1981) reported that the general advice given to pregnant women is that they can safely continue their accustomed level of exercise but should not undertake a new strenuous activity. This suggests that a new, unaccustomed activity might jeopardise the delicate maternal-fetal relationship. The results of the present study show that in rats, mild aerobic exercise does not affect fetal outcome. This is not to say that higher intensified levels of maternal exercise might not affect fetal outcome for there is a smaller number of fetuses on average for those rats who undertook the exercise, although the actual difference is not significant. Both the PR and PC groups followed the same routine up to and including the mating period and there is no reason to suggest that either group would have more or less fetuses. It would be interesting to observe the effects of increased levels of maternal exercise on the number of fetuses as our results suggest this variable might be affected.

The acclimatisation schedule would appear to have had no effect on the development of the mothers in both the PC and PR groups for the line in Figure 1 showing weight gain of the non-pregnant running group is almost indistinguishable from the control group of normal animals.

Several investigators have reported that fetal heart rate (FHR) increases during maternal exercise, but more recently fetal breathing movements (FBM) have been shown to be more sensitive (Marsal et al, 1979). Manning (1977) also reported that FBM are primarily diaphragmatic in nature, as is breathing in the newborn. It was for these reasons that the developing diaphragm was selected for study as representative of the respiratory system. No significant differences in development of the diaphragm muscle could be demonstrated between the PR and PC groups although some variation did exist.
The histological and histochemical development of fetal skeletal muscle as described by Ashmore et al (1972) which was used as our developmental model also requires further investigation because it might not be sufficiently precise for our needs. Around the time of birth there are many changes taking place in the fetal skeletal muscle as it matures (Ashmore et al, 1972) and as such it is an ideal time to examine differences in maturity. The relationship between these changes and their progress needs to be more clearly defined perhaps by the production of a more detailed classification system.

Although the diaphragm was used in this study as representative of the developing skeletal muscle, other areas of muscle are also suggested for investigation. The diaphragm is a complex muscle being derived embryologically from the septum transversum, the body wall and the dorsal mesogastrium. It is conceivable that these three origins develop independently and although every attempt was made to collect each sample from the portion originating from the body wall, this might not always have been possible as the fetal diaphragm is small. The diaphragm is essential to neonatal breathing because the enlarged liver has reduced the ability of the intercostal muscles to act effectively upon the thoracic cage. It is possible that the diaphragm might be given preferential treatment during fetal life in times of nutritional deprivation in a manner similar to that of the heart, brain and liver. Changes in development of the diaphragmatic muscle might only occur in conditions of extreme deprivation. Other muscles might manifest more readily any changes in maturation following nutritional deprivation. These muscles might be found in the caudal end of the fetus as fetal blood distribution is "selective" with blood having the greatest oxygen content being directed through the foramen ovale to the arch of the aorta where it supplies the head and brain in particular, as well as the heart itself. The blood with the lowest oxygen content is directed from the superior vena cava to the right ventricle and along the ductus arteriosus to supply the caudal end of the fetus. Perhaps a better muscle for study would be a muscle of the hindleg which might demonstrate more readily any maturational changes following maternal exercise.

Other workers have shown that maternal exercise directly affects the fetus by increasing its movements (Marsal et al, 1979) and the by-products of maternal exercise also appear to be capable of affecting fetal development by changing the fetal environment either chemically (nutritionally) and/or physically (reduced blood flow). It is perhaps reassuring and not surprising that the results of the present experiment with its mild workload have shown no major developmental changes, indicating perhaps that maternal-fetal mechanisms can adapt to the induced alterations with few long-term consequences. It is conceivable that the small number of 
animals in this study has camouflaged possible slight differences due to exercise that might only become manifest with a larger series of animals. The experiment however, has demonstrated a possible baseline for maternal activity during gestation and indicated several areas that warrant further investigation. It is now our intention, as the next stage in our series of experiments, to increase the maternal workload and attempt to find a level at which the adaptation mechanisms break down inducing fetal changes.

\section{REFERENCES}

Ashmore, C., Robinson, W., Rattray, P. and Doerr, L., 1972 "Biphasic development of muscle fibres in fetal lamb". Experimental Neurol. 37: 241-255.

Clapp, J. F., 1980 "Acute exercise stress in the pregnant ewe". Amer.J.Obstet.Gynecol. 136: 489-494.

Dohm, G. and Beecher, G., 1981 "The ovariectomised female rat as a model animal for the study of adaptation to endurance training". Lab.An.Sci. 31: 146-148.

Gennser, G., Marsal, K. and Brantmark, B., 1976 "Maternal smoking and fetal breathing movements". Am.J.Obstet. Gynecol. 123: 861.

Gettinger, A., Roberts, A. and Campbell, S., 1978 "Comparison between subjective and ultrasound assessments of fetal movements". BMJ 2: 88-90.

Guth, L. and Samaha, F. J., 1969 "Qualitative differences between actomyosin ATPase of slow and fast mammalian muscle". Experimental Neurol. 25: 138-152.

Lewis, P. J. and Boylan, P., 1979 "Alcohol and fetal breathing". The Lancet Feb. 17 pp. 388 (letter).

Manning, F. A., 1977 "Fetal breathing movements as a reflection of fetal status". Postgrad.Med. 61 (4): 116-121.

Marsal, K., Gennser, G. and Lofgren, O., 1979 "Effects on fetal breathing movements of maternal challenges crossover study on dynamic work, static work, passive movements, hyperventilation and hyperoxygenation". Acta Obstet. Gynecol.Scan. 58 (4): 335-342.

Parizkova, J., 1975 "Impact of daily work-load during pregnancy on lipid metabolism in the liver of the offspring". Europ.J.Appl.Physiol. 34: 323-326.

Patrick, J., 1977 "Measurement of human fetal breathing movements". In: Breathing in the Fetus and the Newborn. Mead Johnson Symposium on Perinatal and Developmental Medicine No. 12.

Pomerance, J. J., Gluck, L. and Lynch, V. A., 1974 "Maternal exercise as a screening test for uteroplacental insufficiency". Obstet.Gynecol. 44: 383.

Wirsen, C. and Larsson, K., 1965 "Histochemical differentiation of skeletal muscle in fetal and newborn mice". J.Emb. Exp.Morph. 12: 759-767.

Woodward, S., 1981 "How does strenuous maternal exercise affect the fetus? A review". Birth and the Family 8: 17-24. 